\title{
About renormalization of the Yang - Mills theory and the ap- proach to calculation of the heat kernel
}

\author{
Aleksandr Ivanov ${ }^{1,2, \star}$ \\ ${ }^{1}$ Department of Mathematics and Mathematical Physics, Physics Faculty of St. Petersburg State University \\ ${ }^{2}$ St. Petersburg Department of V.A. Steklov Institute of Mathematics of the Russian Academy of Sciences \\ 27 Fontanka, St. Petersburg, Russia
}

\begin{abstract}
The quantum theory of Yang - Mills in four-dimensional space - time plays an important role in modern theoretical physics. Currently, this model contains many open problems, therefore, it is of great interest to mathematicians. This work consists of several parts, however, it only offers a new approach and, therefore, it is methodological. First of all, the diagram technique and the mathematical basis will be recalled in the first part. Then the process of renormalization will be explained. It is based on momentum cut-off regularization and described in [1] and [2]. However, this type of the regularization has several problems, as a result, only the first correction is calculated. After common constructions and observations, the first correction will be described in detail. Namely, the heat kernel will be considered since it plays a main role in this formalism. In particular, the method for calculating of coefficients of arbitrary order will be proposed.
\end{abstract}

\section{Introduction to Yang - Mills theory}

Suppose $G$ is a compact group of charges, $\mathfrak{b}$ is the Lie algebra. Assume that $t^{a}$ are generators of the Lie algebra and $f^{a b c}$ are structural constants, such that the equalities

$$
\left[t^{a}, t^{b}\right]=f^{a b c} t^{c}
$$

are fulfilled. Further, the Killing form $\operatorname{tr}[\cdot, \cdot]$ may be introduced on the Lie algebra with the normalization conditions

$$
\operatorname{tr}\left[t^{a}, t^{b}\right]=\mathrm{C}(\mathrm{N}) \delta^{a b},
$$

where $N$ is the number of generators. It is convenient to operate with space $\mathbb{R}^{4}$, where $x^{\mu}, \mu=1, \ldots, 4$ are notation of coordinates.

The Yang - Mills field is a 1-form and has view in local coordinates

$$
A(x)=A_{\mu}^{a}(x) t^{a} d x^{\mu}
$$

In turn, the 2-form

$$
F=F_{\mu \nu}^{a} t^{a} d x^{\mu} \wedge d x^{v}
$$

^e-mail: regul1@mail.ru 
plays a role of the curvature, where

$$
F_{\mu \nu}^{a}=\partial_{\mu} A_{\nu}^{a}-\partial_{\nu} A_{\mu}^{a}+f^{a b c} A_{\mu}^{b} A_{\nu}^{c} .
$$

Based on the constructions (1) and (2), the classical action for the Yang - Mills theory has the form

$$
S(A)=\frac{1}{\alpha} \int \operatorname{tr}\left[F \wedge F^{*}\right]
$$

where $\alpha$ is the dimensionless coupling constant. Two differential operators can be introduced:

$$
\begin{gathered}
M_{1}=\nabla_{\sigma} \nabla^{\sigma} \delta_{\mu \nu}+2\left[F_{\mu \nu}, \cdot\right], \\
M_{0}=\nabla_{\mu} \nabla^{\mu},
\end{gathered}
$$

where

$$
D_{\mu}=\partial_{\mu}+\left[B_{\mu}, \cdot\right] .
$$

Following the formalism of the background field after shifting

$$
A_{\mu}(x)=B_{\mu}(x)+\frac{1}{2} \sqrt{\alpha} a_{\mu}(x)
$$

the effective action can be presented in the form

$$
W(B)=\frac{1}{\alpha} W_{-1}(B)+\sum_{k=0}^{\infty} \alpha^{k} W_{k}(B)
$$

where the first and the second corrections are

$$
\begin{gathered}
W_{-1}(B)=\int \operatorname{tr}\left[F_{\mu v} F^{\mu v}\right] d^{4} x, \\
W_{0}(B)=-\frac{1}{2} \ln \operatorname{det} M_{1}+\ln \operatorname{det} M_{0},
\end{gathered}
$$

and corrections $W_{n}$ for $n>0$ are related to the strongly connected vacuum corrections with $n+1$ loops.

\section{Approach to renormalization}

In the paper [2] a scenario was proposed. If momentum cut-off regularization $\Lambda$ will be introduced then the expansion terms for the effective action (6) will have the form

$$
\begin{aligned}
& W_{0}=W_{0,0}+L W_{0,1} \\
& W_{i}=\sum_{k=0}^{i} L^{k} W_{i, k}, \quad i \geqslant 1
\end{aligned}
$$

where $L=\log \frac{\Lambda}{\mu}$. In this case the problem of the renormalization can be formulated in the form of three conditions:

$$
W_{\text {reg }}(\alpha(\Lambda), \Lambda, \mu)=\frac{1}{\alpha(\Lambda)} W_{-1}+L W_{0,1}+\sum_{n=0}^{\infty} \sum_{k=0}^{n} W_{n, k} \alpha^{n}(\Lambda) L^{k}
$$




$$
\begin{gathered}
\Lambda \frac{d \alpha(\Lambda)}{d \Lambda}=\beta(\alpha(\Lambda)), \\
\frac{d W_{r e g}(\alpha(\Lambda), \Lambda, \mu)}{d \Lambda}=0,
\end{gathered}
$$

where

$$
\beta(x)=\sum_{i=0}^{\infty} \beta_{i+1} x^{i+2} .
$$

The first equality is connected with the structure of the effective action after regularization. The second condition is the Gell-Mann-Low equation for the coupling constant and the third one is condition of renormalizability. It is interesting that this problem is mathematically correct and after some calculations the answer has the form for the new coupling constant

$$
\alpha_{r}(\mu)=\left.e^{-L \beta(\alpha) \partial_{\alpha}} \alpha\right|_{\alpha=\alpha(\Lambda)},
$$

and for the renormalized action

$$
W_{r e n}\left(\alpha_{r}(\mu), \mu\right)=\left.e^{-L \beta(\alpha) \partial_{\alpha}}\left(\sum_{n=-1}^{\infty} W_{n, 0} \alpha^{n}\right)\right|_{\alpha=\alpha(\Lambda)} .
$$

The decision also contains an equality for the coefficients $W_{m, n}$. Namely, the coefficients $W_{m, n}, m \geqslant$ $1, n=1 \ldots m$ can be expressed through the coefficients $W_{-1}$ and $W_{i, 0}$ and the coefficients of the $\beta$-function.

\section{Heat kernel}

The scenario which is described in chapter 2 has some problems. For example, only the first correction is calculated because momentum cut-off regularization needs improvement. But now it is possible to discuss the logarithm of the determinant for (3) and (4).

Assume that $A_{0}$ is the Laplace operator and $A_{1}$ is the covariant Laplace operator, where the covariant derivative is equal to (5). Using the method of proper time [3] it is affordable to write

$$
\operatorname{det} A_{1} / A_{0}=-\int_{0}^{\infty} \frac{d \tau}{\tau} \operatorname{tr}\left(e^{-A_{1} \tau}-e^{-A_{0} \tau}\right)
$$

where $e^{-A_{1} \tau}$ and $e^{-A_{0} \tau}$ are operators with kernels $K_{1}(x, y ; \tau)$ and $K_{0}(x, y ; \tau)$ respectively, which satisfy the problem

$$
\left\{\begin{array}{l}
\left(\frac{\partial}{\partial \tau}+A_{j}\right) K_{j}(x, y ; \tau)=0 \\
K_{j}(x, y ; 0)=\delta(x-y)
\end{array}\right.
$$

for $j=0,1$. Usually, the ansatz for $K_{1}(x, y ; \tau)$ has the form

$$
K_{1}(x, y ; \tau)=K_{0}(x, y ; \tau) \sum_{n=0}^{\infty} \tau^{n} a_{n}(x, y),
$$

and after substitution (9) in (8), the relations for coefficients $a_{n}(x, y)$ have the forms 


$$
\begin{gathered}
(x-y)^{\mu} D_{\mu} a_{0}(x, y)=0, \\
\left((n+1)+(x-y)^{\mu} D_{\mu}\right) a_{n+1}(x, y)=A_{1} a_{n}(x, y) .
\end{gathered}
$$

Usually it is very difficult to calculate all coefficients and find the formula for the heat kernel (9). However, in some special cases it is possible. In some models it is possible to consider the case of covariant constant field (for example in [4]):

$$
D_{\mu} F_{v \rho}(x)=0, \forall \mu, v, \rho, x \in \mathbb{R}^{4} .
$$

But in the case of the Fock-Schwinger calibration

$$
x_{\mu} B_{\mu}(x)=0, \forall x \in \mathbb{R}^{4}
$$

the answer for the gauge field has the form

$$
B_{\mu}(x)=\frac{1}{2} x_{\rho} F_{\rho \mu}(x), \forall x \in \mathbb{R}^{4} .
$$

After the calculations it is not difficult to obtain the answer for the heat kernel

$$
K_{1}(x, y ; \tau)=\frac{1}{(4 \pi \tau)^{d / 2}} \exp \left\{\frac{1}{2} x_{\mu} F_{\mu \nu} y_{\nu}-\frac{1}{4 \tau}(x-y)_{\mu} F_{\mu \sigma} \tau(\operatorname{coth} F \tau)_{\sigma \nu}(\tau)(x-y)_{v}+L(\tau)\right\},
$$

where

$$
L(\tau)=-\frac{1}{2} \ln \left(F_{\mu \nu}^{-1} \tau^{-1}(\sinh F \tau)_{\mu \nu}\right)
$$

\section{General case of heat kernel}

\subsection{Path-ordered exponential}

The main purpose of this chapter is to refuse the constraints for the background fields and to learn how to obtain the coefficients of the heat kernel. In this case it is necessary to remember the definition for the path-ordered exponential

$$
\Phi(x, y):=1+\sum_{n=1}^{\infty}(-1)^{n} \int_{0}^{1} \int_{0}^{1} \ldots \int_{0}^{1} d s_{1} \ldots d s_{n} \frac{d z_{1}^{v_{1}}}{d s_{1}} \ldots \frac{d z_{n}^{v_{n}}}{d s_{n}} B_{v_{1}}\left(z_{1}\right) \ldots B_{v_{n}}\left(z_{n}\right)
$$

where parametrization is by line. It is essential object because it satisfies the differential equation with the boundary condition

$$
(x-y)^{\mu} D_{\mu} \Phi(x, y)=0, \Phi(x, x)=1 .
$$

But how to use this object? For this purpose, let's look at the formula

$$
D_{\mu} \Phi(x, y)=\int_{0}^{1} d s s \frac{d z_{v}}{d s} \Phi(x, z) F_{v, \mu}(z) \Phi(z, y)
$$

which was proved in the article [5]. 


\subsection{Main idea}

Suppose that our goal is to calculate the coefficient $a_{n}(x, y)$ with the arguments $y=x$. To do this, we must apply the composition of the integration operator and the covariant Laplace operator $-D_{\mu} D_{\mu}$ to the path-ordered exponential $\Phi(x, y)$ and then take the point $y=x$.

It's very interesting to remember the formula (11) because after taking $y=x$ :

- $\Phi(x, y) \stackrel{y \longrightarrow x}{\longrightarrow} 1$,

- the formula contains only the tension $F(x)$ and its derivatives,

- the formula contains the numerical coefficients in the form $\int_{0}^{1} d s s^{p}$.

The first aim is to construct the diagram technique, one element of which is the path-ordered exponential

$$
\Phi(x, y)=x \longrightarrow y
$$

and when $y \longrightarrow x$ then a line goes to a dot and one element is related to the tension and its derivatives.

The second aim is to control the numerical coefficients and the degree of parameterization parameters. In this case, it is possible to calculate the coefficients and to do mathematical estimates of the convergence of the series.

\section{Acknowledgments}

This research is supported by a grant from the Russian Science Foundation (Project No. 14-11-00598).

\section{References}

[1] L. D. Faddeev, Int. J. Mod. Phys. A 31, 1630001 (2016)

[2] S. E. Derkachev, A. V. Ivanov, L. D. Faddeev, Theoret. and Math. Phys. 192:2, 1134 (2017)

[3] V. Fock, Phys. Z. Sowjetunion 12, 404 (1937)

[4] J. P. Bornsen, A. E. M. van de Ven, Nucl. Phys. B 657, 257 (2003)

[5] Graham M. Shore, Annals of physics 137, 262 (1981) 